without any discomfort to the patient, except for the bending of her head, the time taken being a few seconds for the appearance and for the disappearance rather longer.

It is also difficult for me to discuss the case, as I have not previously seen, or heard of, a similar one, but as regards the dimness of sight, of which she mainly complains, I am inclined to think that this is due to the heaping up of the "fluid" as it passes over the concave edge of the iris, and this would naturally be more pronounced when the pupil is contracted in a strong light, and in any case her pupils are naturally getting smaller as she gets older. As regards the aetiology of the condition, I can only suppose that this is a form of the rare kind of cyst described by J. Herbert Parsons as being formed between the retinal layers of the iris, but he does not mention that any of these cysts have the peculiar faculty of appearing in the anterior chamber.

\title{
ACUTE ANTERIOR ETHMOIDITIS IN YOUNG SUBJECTS
}

\author{
BY \\ P. A. HARRY, M.D. \\ OPHTHALMIC SURGEON TO THE ROCHDALE INFIRMARY
}

IN reference to the paper by Mr. Sydney Stephenson on acute anterior ethmoiditis in the August number of the BRITISH JOURNAL OF OPHTHALMOLOGY I may say that I have seen cases such as he describes in children of from 4 to 12 years, but the one I am about to relate is of special interest owing to the age of the patient, namely, 3 weeks :

Ernest P. was sent to the Rochdale Infirmary by Dr. S. B. Brentnall on July 9, 1918. The history was that the left eye had been slightly inflamed since birth. The appearances were those of "a cold in the eye." The condition was treated as an instance of ophthalmia neonatorum for the first ten days.

When I first saw the infant the conditions were as follows: L.E., considerable proptosis and great resistance to pressure backwards. The eye projected directly forwards. Slight inflammation of the conjunctiva accompanied with little discharge. There was a considerable amount of discharge of blood-stained material from the left nostril. The pupillary reactions and the fundus of the left eye were normal. Transillumination through the mouth yielded negative results. A similar remark applies to examination of the nose with Holmes's pharyngoscope. The child was peevish and restless, but there was no rise in temperature and no diarrhoea. 
With regard to treatment gentle curetting of the anterior ethmoidal area through the nose was followed by an escape of pus. Hydrogen peroxide was instilled often into the left nostril. Fomentations were applied to the left eye.

On July 11 the proptosis was much reduced. On July 16 the proptosis had disappeared. There was no abnormal orbital resistance. The fundus was normal. Discharge from the left nostril was still copious. On August 8, the child was discharged as cured.

\section{COUNCIL OF BRITISH OPHTHALMOLOGISTS}

\section{Report on the desirability of a special qualification in ophthalmology}

THIS report is divided into five parts :

I.-The existing conditions concerning examinations in special subjects.

II.-Examinations in Ophthalmology in the United Kingdom.

III.-The qualifications required by the principal hospitals of the United Kingdom for appointment to the post of Ophthalmic Surgeon.

IV.-The desirability of a special examination in Ophthalmology. V.-Conclusions and recommendations.

\section{I.-The existing conditions concerning examinations in special subjects}

At present there are in the United Kingdom special examinations in Dental Surgery, Public Health, Tropical Medicine and Ophthalmology.

These examinations may be classified in two groups:

(a) Those in which the special subjects form part of a higher examination in medicine or surgery, e.g., Tropical Medicine; State Medicine; Ophthalmic Surgery; Aural, Nasal and Laryngeal Surgery; and Dental Surgery for the M.D. or M.S. of the University of London: Ophthalmology; Aural, Nasal and Laryngeal Surgery; Advanced Midwifery with Obstetric Surgery and Gynaecology; Advanced Anatomy; and Dental Surgery for the Fellowship of the Royal College of Surgeons, Edinburgh.

(b) Those in which qualified medical men are examined in a special subject, independently of any examination in general medicine or surgery, e.g., for the Diploma in Public Health, University of Cambridge; the Licence in Dental Surgery, Royal College of Surgeons, England; and the Diploma in Ophthalmology, University of Oxford. 